\title{
LA AUDITORÍA FORENSE, UNA PERSPECTIVA DESDE LA MALLA CURRICULAR DE LAS CARRERAS DE “CONTABILIDAD Y AUDITORÍA” EN EL ECUADOR
}

THE FORENSIC AUDIT, A PERSPECTIVE FROM THE CURRICULAR

SUBJECTS OF THE "ACCOUNTING AND AUDITING" CAREERS IN ECUADOR

Fecha de recepción: 26 de febrero de 2018.

Fecha de aceptación: 28 de mayo 2018.

Código JEL: M40, M42, M48

Código DOI: 10.25097/rep.n28.2018.02
Teodoro Cubero Abril

tcubero@uazuay.edu.ec

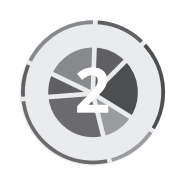

\section{Resumen}

El presente trabajo analiza la importancia de la auditoría forense, desde la óptica de los currículos en las carreras de contabilidad y auditoría en las universidades ecuatorianas.

Los países latinoamericanos presentan frecuentes actos de corrupción e impunidad, que han generado indignación en la sociedad, y que restan recursos destinados a satisfacer necesidades de la colectividad. Por lo antes mencionado, se desprende que, en las carreras de grado y programas de posgrado en contabilidad y auditoría, no incluyen en sus currículos la asignatura auditoría forense con la finalidad de que sus resultados de aprendizaje profesionales respondan a los problemas de la sociedad.

Es pertinente que las carreras de contabilidad y auditoría con la participación de sus futuros profesionales den respuesta, ante los hechos de fraude financiero, que atenta contra el bienestar de la población.

Palabras Clave: Auditoría Forense, fraude, currículo, contabilidad y auditoría, Instituciones de Educación Superior (IES), corrupción.

\section{Abstract}

This paper analyzes the importance of forensic auditing, from the perspective of the curricula in accounting and auditing careers in Ecuadorian universities.

The Latin American countries present frequent acts of corruption and impunity, which have generated discussion in society, and which detract from their limited budgets.In consequence, it follows that in bachelor's and master's degree, accounting and auditing careers, do not include in their curricula the subject of forensic audit in order that their professional learning outcomes, respond to the problems of society.

It is pertinent that accounting and auditing careers respond through the participation of their future professionals, in the face of financial frauds, which threatens the welfare of the population.

Keywords: Forensic Audit, fraud, curriculum, accounting and auditing, Institutions of Higher Education, corruption. 


\section{Introducción}

Distintos autores y organismos de control, han publicado sobre la Auditoría Forense, efectuando importantes propuestas, lo cual evidencia la importancia que esta actividad ha cobrado como un área especializada de la auditoría. Cano y Lugo (2005) mencionan que la auditoría forense nació con el código de Hamurabi, entre los cuales existen normas sobre el trabajo de demostrar con documentos contables un fraude, señalando además, que posiblemente el primer auditor forense fue el funcionario (un contador) que evidenció las actividades mafiosas de AI Capone en 1930 en Estados Unidos, actuación que originó el uso de la auditoría para presentar información ante un tribunal y contribuir a la determinación del veredicto, colaborando en la demostración de un hecho delictuoso y punible.

Los efectos de la corrupción se han convertido en el mayor impedimento para el desarrollo económico, cuyo impacto sobre la inversión, crecimiento y reducción de la pobreza no se puede ignorar (Banco Mundial, 2009). En la actualidad asistimos a un escenario con mayores repercusiones que a no dudarlo han usurpado las oportunidades y la condición económica, especialmente de la población más pobre.

Al respecto, Badillo (2013) define a la auditoría forense como aquella labor que se enfoca en la prevención y detección del fraude financiero; por ello, generalmente los resultados del trabajo del auditor forense son puestos a consideración de la justicia, que se encargará de analizar, juzgar y sentenciar los delitos cometidos (corrupción financiera, pública o privada).

Por consiguiente, el fraude es cualquier acto ilegal caracterizado por engaño, ocultación o violación de la confianza, dichos actos no requieren de amenaza o violencia física. Los fraudes son perpetrados por individuos u organizaciones para obtener dinero, bienes o servicios o para asegurarse ventajas personales o de negocios. (Instituto de Auditores Internos del Ecuador, 2013)

En estos tiempos de constantes transformaciones y redefiniciones, la auditoría ha evolucionado como concepto teórico y como práctica para adaptarse a las exigencias de la globalización y de los avances tecnológicos, organizacionales y comunicacionales, enfrentando los diferentes ambientes donde es utilizada, con un entorno que se caracteriza por ser dinámico y cambiante. La presencia de la corrupción, en sus modalidades más comunes de fraude, maquillaje de estados financieros, lavado de dinero y apropiación indebida de activos, es según Estupiñán (2002) la mayor preocupación en el sector público y privado. En tal virtud, combatir estas maniobras delictivas es uno de los principales desafíos que afronta América Latina en general y el Ecuador en particular.

Al respecto, conscientes de lo que representa la corrupción y sus efectos, diferentes países han suscrito acuerdos y se han creado organismos con la finalidad de combatir el aprovechamiento ilícito del patrimonio público. Por ejemplo La Convención Interamericana contra la Corrupción (CICC) la Organización a los Estados Americanos (OEA-1997), la Convención para Combatir el Cohecho de Servidores Públicos Extranjeros en Transacciones Comerciales Internacionales de la Organización para la Cooperación y el Desarrollo Económico (1999), la Convención de las Naciones Unidas contra la Corrupción (UNCAC, según siglas en inglés, 2005), la Organización Latinoamericana y del Caribe de Entidades Fiscalizadoras Superiores (OLACEFS).

En la última década, el Ecuador ha sido escenario de hechos fraudulentos que han mutilado el erario nacional y la gestión pública, circunstancia que brinda una especial oportunidad para el desarrollo de la auditoría forense, de implantar estrategias de control antifraude y programas contra la corrupción.

El riesgo de fraude puede ser reducido a través de una combinación de medidas de prevención y disuasión, que son mucho menos costosas que las requeridas para la detección e investigación de estas desviaciones. Se ha considerado que una herramienta estratégica de prevención de fraudes puede ser la ejecución de auditorías forenses. Consecuentemente, resulta una opción válida considerar a la auditoría forense como medio para luchar contra la corrupción.

Conviene señalar que al profesional que le corresponde dictaminar sobre la razonabilidad de los saldos de las cuentas de los estados financieros y determinar si existe fraude o error, es el contador público. Sin 
embargo, la mayoría de los currículos de las carrearas de contabilidad y auditoría de las Universidades del Ecuador que ofertan esta profesión, tanto en pregrado como en posgrado no incluyen la materia de auditoría forense, cuyos contenidos promuevan competencias específicas que contribuyan a prevenir, detectar y establecer mecanismos de control antifraude.

La auditoría forense es una alternativa para combatir la corrupción, porque permite que expertos emitan ante los jueces conceptos y opiniones de valor técnico, que le permiten a la justicia actuar con mayor certeza (Rozas, 2009, p. 68). Actualmente la investigación del fraude requiere de habilidades combinadas de un auditor bien entrenado y de un investigador criminal, no existiendo en los contadores ambas características.

En procura de impedir las prácticas deshonestas, surge la auditoría forense, como una modalidad de auditoría que pretende una revisión minuciosa y detallada de las operaciones organizacionales, cuya finalidad es una determinación judicial, ante actos dolosos o fraudulentos, mediante la aplicación de técnicas, procedimientos específicos y normas de auditoría que permitan obtener evidencia de apoyo al Poder Judicial (Castillo, Figueredo y Méndez, 2012).

El objetivo de este trabajo es analizar el avance de la auditoría forense en el Ecuador y la formación en esta materia de profesionales en las Universidades de las carreras de "contabilidad y auditoría", con competencias en la prevención, detección y trámite de los hechos de fraude, desde la perspectiva de sus mallas curriculares.

Este estudio pretende dejar en los lectores especialmente de las carreras de contabilidad y auditoría el anhelo de formar a los futuros profesionales con sólidos conocimientos de auditoría forense como respuesta a los problemas de corrupción y confronte a los fraudes financieros.

\section{Método}

Se revisaron artículos relacionados con la auditoría forense, que plantean el tratamiento y metodología para la ejecución de esta temática como herramienta necesaria en la lucha contra la corrupción. Se utilizaron varias fuentes documentales, tales como publicaciones realizadas por organismos internacionales especializados en el tema, así como estudios académicos e investigaciones para determinar el estado del arte, selección bibliográfica se hizo a base de la experiencia personal en la cátedra, en base a lo cual se preparó fichas de lectura, resaltando, el desarrollo de la auditoría forense frente a los hechos de fraude.

Mediante la revisión de las páginas web de las IES y del CES, se realizó un levantamiento de la información de las mallas curriculares de las universidades que ofertan la carrera de Contabilidad y Auditoría tanto en grado como en posgrado, con el objeto de determinar si se imparte la asignatura auditoría forense, antes y después del año 2016. Año en el cual, se cambió las mallas curriculares con la aprobación de los nuevos proyectos de carrera de grado, que otorga el título de licenciado en contabilidad y auditoría, conforme con la nueva nomenclatura de títulos emitida por el CES. Igual se procedió con los programas de maestría que ofertan las IES en este campo, o que incluyen módulos o asignaturas relacionadas con auditoría forense.

\section{Análisis de literatura y fundamentos}

El fraude y la corrupción no son fenómenos recientes ni es una particularidad de una sociedad específica, se presenta en la mayoría de las civilizaciones antiguas. En la actualidad, la corrupción se ha convertido en el obstáculo para el desarrollo económico. La década de 1990 fue testigo de su surgimiento y desarrollo, cuyo impacto ha generado reducciones en la inversión, crecimiento y en los niveles de pobreza.

En la última década, el escándalo de corrupción que ha estremecido a casi toda Latinoamérica han sido los millonarios casos de soborno realizados por la constructora brasileña Odebretch, en donde se han visto involucrados, altos funcionarios públicos incluyendo ex presidentes, debido a las denuncias realizadas por 
los directivos sindicados de dicha empresa y las fiscalías de muchos países, que se encuentran procesando información que devienen en encarcelamientos y la posibilidad de recuperar los recursos sustraídos.

En la primera mitad del siglo pasado, los auditores realizaban verificaciones exclusivamente contables tales como: activos, constatando inventario, conciliando el disponible, algunas organizaciones priorizaron la labor de los auditores en asegurar el cumplimiento de las disposiciones legales. En algunas ocasiones, los auditores internos se concentraron en investigar fraudes, en lo que hoy se conoce como auditoría forense.

De La Torre (2017) señala que, en la actualidad, auditoría es mucho más que la mera verificación contable, convirtiéndose en un instrumento imprescindible, destinada a generar información vital para que los administradores supervisen y gestionen correctamente la organización, pero también para que los grupos de interés puedan confiar en la buena gestión de la entidad y en la información que emiten al mercado o presentan a los entes reguladores.

Con la finalidad de frenar los hechos de fraude deshonestos, aparece la auditoría forense, cuya aplicación requiere de un proceso metodológico realizando una revisión cuidadosa y detallada de las operaciones públicas o privadas con el objeto de lograr resultados que encaminen la determinación judicial, de hechos fraudulentos, mediante la aplicación de técnicas, procedimientos específicos o alternativos y normas de auditoría que permitan obtener evidencia de apoyo al Poder Judicial (Castillo, Figueredo y Méndez, 2012). "La Auditoria Forense es una alternativa para combatir la corrupción, porque permite que expertos emitan ante los jueces conceptos y opiniones de valor técnico, que le permiten a la justicia actuar con mayor certeza" (Rozas, 2009, p. 68).

Debido al crecimiento de los actos de corrupción, el mundo entero se encuentra en un proceso de estandarización como los de autorregulación, ética empresarial, códigos de conducta, gestión de riesgos de fraude, normas ISO, gobierno corporativo, COSO, etc., conceptos que se encuentran intrínsecamente relacionados (De La Torre, 2017).

La labor del auditor forense no solamente se destaca en las investigaciones en curso, sino en etapas previas al fraude, a base del cual formula recomendaciones para la implantación de mecanismos de control antifraude, de manera que no se vuelvan a presentar en el futuro, como por ejemplo, la presentación de información financiera inexacta por manipulación intencional, falsificación, maquillaje de estados financieros, lavado de activos, etc. (Ocampo, Trejos y Solarte, 2010).

Varios hechos de corrupción en Latinoamérica y particularmente en el Ecuador en el año 2016, han sido los millonarios casos de soborno realizados por la constructora brasileña Odebretch, en donde funcionarios públicos de alta jerarquía, tales como Presidentes, ex presidentes, ex vicepresidentes, ex Ministros de Estado entre los principales, que han sido procesados, otros sentenciados penalmente, debido a denuncias realizadas por los directivos de dicha empresa y las fiscalías de muchos países.

La corrupción, es un fenómeno social, la decisión de realizar actos fraudulentos está en función del contexto social, de sus valores y las interrelaciones sociales. Por lo tanto hablar de corrupción es comprender la dinámica de los lazos y relaciones que se construyen entre los diferentes agentes para que dicha práctica sea posible de realizar e incluso posible de estabilizar Arellano (2017).

En un estudio realizado por la Agencia de los Estados Unidos para el Desarrollo Internacional (USAID 2004) denominado; la Corrupción en América Latina, en lo referente a la rendición de cuentas y anticorrupción en las américas, se señala que los países latinoamericanos comparten algunas características comunes sobre la corrupción y que continúan vigentes, tales como;

\section{Una función pública en transición.}

2. Leyes engorrosas, superposición de responsabilidades de diferentes organismos gubernamentales y confusión sobre las leyes. 
3. Procedimientos burocráticos y reglamentaciones complejas y excesivas.

4. Debilidades de las instituciones de control y fiscalización.

5. Legislaturas inefectivas.

6. Sistemas judiciales disfuncionales que no son eficientes ni independientes.

7. Controles sociales débiles, escasa articulación y falta de voz.

8. Más conciencia, pero actitudes mixtas hacia la corrupción.

9. Ausencia de voluntad política para controlar la corrupción.

Ríos (2014) define a la Auditoría Forense, como un procedimiento técnico-científico que permite que un experto emita ante los jueces conceptos y opiniones de valor técnico, especialmente en lo relativo a la vigilancia de la gestión fiscal, financiera y monetaria de una organización bien sea pública o privada; de esta manera, se contribuye a mejorar las economías de los países.

(Díaz Ortega \& Perez Peña, 2015) Existen dos tipos de enfoques que coadyuvan a determinar cuál será el plan de acción en una organización para el tratamiento del problema de fraude, y podemos definirlos como la auditoría forense preventiva y la auditoría forense detectiva.

(Arango Aguirre, 2012) El auditor forense debe ser un profesional altamente capacitado, experto conocedor de: contabilidad, auditoría, control interno, administración de riesgos, tributación, finanzas, informática, técnicas de investigación, legislación penal y otras disciplinas. En cuanto a su formación como persona el auditor forense debe ser objetivo, independiente, justo, honesto, inteligente, analítico, astuto, sagaz, planificador, prudente y precavido.

En el Ecuador el Código Orgánico Integral Penal sustenta la auditoría forense y establece el marco de ejecución del juzgamiento y sanción. Uno de los aspectos que caracteriza a la auditoria forense se fundamenta en la observancia de las normas internacionales de auditoría y aseguramiento, y en las normas y procedimientos expedidos por la Contraloría General del Estado para los hechos de fraude en el sector público. Estas normas de auditoría constituyen los estándares mínimos que deben observarse en el trabajo de auditoría, relacionadas no solo con los atributos personales del auditor, sino de carácter técnico tales como el trabajo de campo y la formulación del informe de resultados.

Por consiguiente, la auditoría forense es un medio para combatir la corrupción, constituye un importante modelo de control y de investigación, que ayuda a detectar y combatir los delitos cometidos. La población ecuatoriana ha sido testigo de la proliferación de los escándalos financieros y fraudes, en los últimos años, que ha generado preocupación en la sociedad, lo cual demanda la participación reiterada de los auditores forenses en los procesos judiciales.

La contabilidad y la auditoría como profesión con responsabilidad social, es esencial para asegurar el bienestar de los miembros de una sociedad, se basan en preparación técnica y científica, normas y comportamientos éticos, a los cuales están obligados quienes la ejercen.

En este marco la participación de las universidades en la formación de profesionales en la carrera de contabilidad y auditoría, juega un papel fundamental en la lucha contra la corrupción mediante la auditoría forense.

En la formación académica de los profesionales de la carrera de contabilidad y auditoría, impartida en las universidades del país, hasta antes del año 2016, casi no se ha considerado en los currículos de la carrera una materia especializada en auditoría forense, y en muchas ocasiones ni siquiera está incluida como temática a ser impartida dentro de otras materias relativas a la auditoría; por lo cual los profesionales graduados y sin experiencia en el campo, carecen de las competencias necesarias para desarrollar este trabajo.

De igual manera quienes dirigen las empresas normalmente tienen desconocimiento de la conveniencia 
de contar con una herramienta como la auditoría forense para contrarrestar el fraude y que podría inciden negativamente en la consecución de los objetivos organizacionales.

\section{Resultados}

Con la información de las páginas web del CES y de las Universidades acreditadas en el Ecuador, referente a la inclusión de la materia de auditoría forense en los currículos de la carrera de contabilidad y auditoría, se obtiene los siguientes resultados:

Tabla 1. Universidades que ofertan la carrera de contabilidad y auditoría (marzo 2017 pregrado)

\begin{tabular}{|c|c|c|c|c|}
\hline UNIVERSIDAD & CATEGORÍA & PRESENCIAL & SEMIPRESENCIAL & A DISTANCIA \\
\hline ESCUE. SUPERIOR POLITECNICA DE CHIMBORAZO & $\mathrm{B}$ & $x$ & & \\
\hline PUCE & B & $\mathrm{x}$ & & \\
\hline UCACUE & B & $\mathrm{x}$ & & $\mathrm{x}$ \\
\hline UNIVERSIDAD CENTRAL DEL ECUADOR & $\mathrm{B}$ & $\mathrm{x}$ & & $\mathrm{x}$ \\
\hline UNIVESIDAD DE CUENCA & A & $\mathrm{x}$ & & \\
\hline UNIVERSIDAD DEL AZUAY & B & $\mathrm{x}$ & & \\
\hline UNIVERSIDAD ESTATAL DE BOLIVAR & C & $\mathrm{x}$ & & \\
\hline UNIVERSIDAD ESTATAL SUR DE MANABI & C & $\mathrm{x}$ & & \\
\hline UNIVERSIDAD ESTATAL PENÍNSULA DE SANTA ELENA & $\mathrm{C}$ & $\mathrm{x}$ & & \\
\hline UNIVERSIDAD LAICA ELOY ALFARO DE MANABI & $\mathrm{C}$ & $\mathrm{x}$ & & \\
\hline UNIVERSIDAD LAICA VIENTE ROCAFUERTE & $\mathrm{C}$ & $\mathrm{x}$ & & \\
\hline UNIVERSIDAD NACIONAL DE CHIMBORAZO & $\mathrm{C}$ & $\mathrm{x}$ & & \\
\hline UNIVERISIDAD NACIONAL DE LOJA & B & $\mathrm{x}$ & & $x$ \\
\hline UEES & A & $\mathrm{x}$ & & \\
\hline UNIVERSIDAD POLITÉCNICA SALESIANA & B & $\mathrm{x}$ & & \\
\hline UNIVERSIDAD TÉCNICA DE AMBATO & $\mathrm{B}$ & $\mathrm{x}$ & $\mathrm{x}$ & \\
\hline UNIVERSIDAD TÉNCIA DE BABAHOYO & $\mathrm{C}$ & $\mathrm{x}$ & & \\
\hline UNIVERSIDAD TÉCNICA DE COTOPAXI & C & $\mathrm{x}$ & & \\
\hline UNIVERSIDAD TÉCNICA DE MACHALA & C & $x$ & & \\
\hline UNIVERSIDAD TÉCNICA DE MANABI & B & $\mathrm{x}$ & & \\
\hline UNIVERSIDAD LUIS VARGAS TORRES DE ESMERALDAS & C & $\mathrm{x}$ & & \\
\hline UTPL & B & $\mathrm{x}$ & & $x$ \\
\hline UNIVERSIDAD TECNOLÓGICA EMPRESARIAL DE GUAY. & B & $\mathrm{x}$ & & \\
\hline UNIVERSIDAD TENOLÓGICA IBEROAMERICA & B & $\mathrm{x}$ & & $\mathrm{x}$ \\
\hline UNIVERESIDAD TECNOLÓGICA ISRAEL & $\mathrm{C}$ & $\mathrm{x}$ & $x$ & $\mathrm{x}$ \\
\hline UNIVERSIDAD CATÓLICA SANTIAGO DE GUAYAQUIL & B & & & $x$ \\
\hline UNIVERSIDAD INTERNACIONAL DEL ECUADOR & B & & & $\mathrm{x}$ \\
\hline ESPE & $\mathrm{A}$ & & $\mathrm{x}$ & \\
\hline TOTAL & 28 & 25 & 3 & 8 \\
\hline PORCENTAJE & $100 \%$ & $89 \%$ & $10 \%$ & $29 \%$ \\
\hline
\end{tabular}

Fuente: Elaboración propia con base en datos del CES (2018)

El Ecuador cuenta con 55 IES, acreditados en tres categorías (A; B y C) de las cuales 28, ofertan carreras de contabilidad y auditoría que representa el $51 \%$ de las universidades, llevando a cabo su desarrollo 
académico mediante las modalidades; presencial, semipresencial y a distancia.

Tabla 2. Universidades que ofertan la carrera de contabilidad y auditoría y cuentan en su malla curricular con la asignatura de auditoría forense

\begin{tabular}{|c|c|c|c|c|}
\hline \multirow[b]{2}{*}{ UNIVERSIDAD } & \multicolumn{2}{|c|}{ ANTES DEL AÑo 2016} & \multicolumn{2}{|c|}{ DESPUES DEL AÑo 2016} \\
\hline & SI & No & SI & No \\
\hline ESCUE. SUPERIOR POLITECNICA DE CHIMBORAZO & & $\mathrm{x}$ & $\mathrm{x}$ & \\
\hline PUCE & & $\mathrm{x}$ & & $\mathrm{x}$ \\
\hline UCACUE & & $\mathrm{x}$ & & $\mathrm{x}$ \\
\hline UNIVERSIDAD CENTRAL DEL ECUADOR & & $\mathrm{x}$ & & $\mathrm{x}$ \\
\hline UNIVESIDAD DE CUENCA & $\mathrm{x}$ & & $\mathrm{x}$ & \\
\hline UNIVERSIDAD DEL AZUAY & $\mathrm{x}$ & & $\mathrm{x}$ & \\
\hline UNIVERSIDAD ESTATAL DE BOLIVAR & & $\mathrm{x}$ & $\mathrm{x}$ & \\
\hline UNIVERSIDAD ESTATAL SUR DE MANABI & & $\mathrm{x}$ & & $\mathrm{x}$ \\
\hline UNIVERSIDAD ESTATAL PENÍNSULA DE SANTA ELENA & & $\mathrm{x}$ & $\mathrm{x}$ & \\
\hline UNIVERSIDAD LAICA ELOY ALFARO DE MANABI & & $\mathrm{x}$ & $\mathrm{x}$ & \\
\hline UNIVERSIDAD LAICA VIENTE ROCAFUERTE & & $\mathrm{x}$ & $\mathrm{x}$ & \\
\hline UNIVERSIDAD NACIONAL DE CHIMBORAZO & & $\mathrm{x}$ & & $\mathrm{x}$ \\
\hline UNIVERISIDAD NACIONAL DE LOJA & & $\mathrm{x}$ & & $\mathrm{x}$ \\
\hline UEES & & $\mathrm{x}$ & & \\
\hline UNIVERSIDAD POLITÉCNICA SALESIANA & & $\mathrm{x}$ & & $\mathrm{x}$ \\
\hline UNIVERSIDAD TÉCNICA DE AMBATO & & $\mathrm{x}$ & $\mathrm{x}$ & \\
\hline UNIVERSIDAD TÉNCIA DE BABAHOYO & & $\mathrm{x}$ & & $\mathrm{x}$ \\
\hline UNIVERSIDAD TÉCNICA DE COTOPAXI & & $\mathrm{x}$ & $\mathrm{x}$ & \\
\hline UNIVERSIDAD TÉCNICA DE MACHALA & & $\mathrm{x}$ & $\mathrm{x}$ & \\
\hline UNIVERSIDAD TÉCNICA DE MANABI & & $\mathrm{x}$ & $\mathrm{x}$ & \\
\hline UNIVERSIDAD LUIS VARGAS TORRES DE ESMERALDAS & & $\mathrm{x}$ & & $\mathrm{x}$ \\
\hline UTPL & & $\mathrm{x}$ & & $\mathrm{x}$ \\
\hline UNIVERSIDAD TECNOLÓGICA EMPRESARIAL GUAYA. & & $\mathrm{x}$ & $\mathrm{x}$ & \\
\hline UNIVERSIDAD TENOLÓGICA IBEROAMERICA & & $\mathrm{x}$ & & $\mathrm{x}$ \\
\hline UNIVERESIDAD TECNOLÓGICA ISRAEL & & $\mathrm{x}$ & $\mathrm{x}$ & \\
\hline UNIVERSIDAD CATÓLICA SANTIAGO DE GUAYAQUIL & & $\mathrm{x}$ & $\mathrm{x}$ & \\
\hline UNIVERSIDAD INTERNACIONAL DEL ECUADOR & & $\mathrm{x}$ & $\mathrm{x}$ & \\
\hline ESPE & & $\mathrm{x}$ & & $\mathrm{x}$ \\
\hline TOTAL & 2 & 26 & 16 & 12 \\
\hline PORCENTAJE & $\mathbf{7 \%}$ & $93 \%$ & $\mathbf{5 7 \%}$ & $42 \%$ \\
\hline
\end{tabular}

Fuente: Elaboración propia con base en datos del CES (2018)

Se puede evidenciar, que del total de carreras que ofertan contabilidad y auditoría, antes del 2016, solamente el $7 \%$ incluyen la asignatura de auditoría forense en sus mallas curriculares en tanto que después del año 2016 , el $57 \%$ de los programas curriculares en esta carrera, introducen dicha materia. 
Tabla 3. Universidades de posgrado que ofertan maestrías en contabilidad y auditoría

\begin{tabular}{l|ccc}
\hline UNIVERSIDAD & CATEGORÍA & SI OFERTAN & NO OFERTAN \\
\hline IAEN & $\mathrm{B}$ & 0 & $\mathrm{X}$ \\
\cline { 2 - 4 } FLACSO & $\mathrm{A}$ & 0 & $\mathrm{X}$ \\
\hline UNIVERSIDAD ANDINA SIMON BOLIVAR & $\mathrm{A}$ & 0 & $\mathrm{X}$ \\
\hline TOTAL & 3 & 0 & 3 \\
\hline PORCENTAJE & $100 \%$ & $0 \%$ & $100 \%$ \\
\hline
\end{tabular}

Fuente: Elaboración propia con base en datos del CES (2018)

El sistema de educación superior en el Ecuador cuenta con tres IES de posgrado, sin embargo no se han desarrollado programas de posgrado en auditoría forense o que incorporen esta materia o módulo, en tales programas.

Tabla 4. Universidades que ofertan maestrías que incluyen la asignatura de auditoría forense

\begin{tabular}{|c|c|c|c|c|c|}
\hline \multirow[t]{2}{*}{ UNIVERSIDAD } & \multirow[t]{2}{*}{ CATEGORÍA } & \multicolumn{2}{|c|}{ ANTES DEL AÑO 2016} & \multicolumn{2}{|c|}{ DESPUES DEL AÑO 2016} \\
\hline & & $\mathrm{SI}$ & NO & $\mathrm{SI}$ & NO \\
\hline PUCE & $\mathrm{B}$ & & & & \\
\hline UCACUE & B & & $\mathrm{x}$ & $\mathrm{x}$ & \\
\hline UNIVERSIDAD CENTRAL DEL ECUADOR & B & & & & \\
\hline UNIVESIDAD DE CUENCA & A & & $\mathrm{x}$ & $\mathrm{x}$ & \\
\hline UNIVERSIDAD DEL AZUAY & $\mathrm{B}$ & $\mathrm{X}$ & & & $\mathrm{x}$ \\
\hline UNIVERSIDAD ESTATAL DE BOLIVAR & C & & & & \\
\hline UNIVERSIDAD ESTATAL SUR DE MANABI & $\mathrm{C}$ & & & & \\
\hline UNIVERSIDAD ESTATAL PENÍNSULA DE SANTA & $\mathrm{C}$ & & & & \\
\hline UNIVERSIDAD LAICA ELOY ALFARO DE MANA & $\mathrm{C}$ & & & & \\
\hline UNIVERSIDAD LAICA VIENTE ROCAFUERTE & C & & & & \\
\hline UNIVERSIDAD NACIONAL DE CHIMBORAZO & C & & & & \\
\hline UNIVERISIDAD NACIONAL DE LOJA & B & & & & \\
\hline UEES & A & & & & \\
\hline UNIVERSIDAD POLITÉCNICA SALESIANA & B & & & & \\
\hline UNIVERSIDAD TÉCNICA DE AMBATO & B & & & & \\
\hline UNIVERSIDAD TÉNCIA DE BABAHOYO & $\mathrm{C}$ & & & & \\
\hline UNIVERSIDAD TÉCNICA DE COTOPAXI & C & & & & \\
\hline UNIVERSIDAD TÉCNICA DE MACHALA & C & & & & \\
\hline UNIVERSIDAD TÉCNICA DE MANABI & B & & & & \\
\hline UNIVERSIDAD LUIS VARGAS TORRES DE ESME & $\mathrm{C}$ & & & & \\
\hline UTPL & $\mathrm{B}$ & & & & \\
\hline UNIVERSIDAD TECNOLÓGICA EMPRESARIAL [ & B & & & & \\
\hline UNIVERSIDAD TENOLÓGICA IBEROAMERICA & B & & & & \\
\hline UNIVERESIDAD TECNOLÓGICA ISRAEL & C & & & & \\
\hline UNIVERSIDAD CATÓLICA SANTIAGO DE GUAY & B & & & & \\
\hline UNIVERSIDAD INTERNACIONAL DEL ECUADOI & B & & & & \\
\hline ESPE & A & & & & \\
\hline TOTAL & 28 & 1 & 2 & 2 & 1 \\
\hline PORCENTAJE & $100 \%$ & $3.6 \%$ & $7 \%$ & $7 \%$ & $3.6 \%$ \\
\hline
\end{tabular}

Fuente: Elaboración propia con base en datos del CES (2018) 
De las universidades que ofertan la carrera en contabilidad y auditoría (28) además del programa de pregrado, han desarrollado programas de posgrado en contabilidad y auditoría, el 10,65 de las IES antes y después del año 2016. Incorporando en igual porcentaje la asignatura auditoría forense.

\section{Discusión}

En el Ecuador hay pocas investigaciones relacionadas con la auditoría forense, a pesar de que en los últimos años se han incrementado los niveles de corrupción en el sector público y privado, motivo por el cual resulta necesario investigar actividades ilícitas tales como: lavado de activos; fraudes; contabilidad fraudulenta; evasión tributaria, asociaciones para ocultar deudas; sobornos como en el caso de la Empresa Brasileña Odebrecht; fraudes en empresas y sector público del Ecuador; convirtiéndose en un elemento de evaluación, es ahí donde los profesionales especializados en auditoría forense, cumplan un rol fundamental para evaluar actividades que perjudican a la sociedad ecuatoriana.

En los pocos trabajos de investigación sobre la auditoría forense en el Ecuador, no se ha considerado la oferta académica en grado y posgrado de las IES, que transmitan conocimientos sobre auditoría forense, como una asignatura específica a los futuros profesionales.

Existen varias IES que ofertan la carrera de contabilidad y auditoría en pregrado, a partir del año 2016 (51\% de las IES), especialmente de manera presencial, evidenciándose un número considerable de profesionales a disposición de la sociedad.

Una vez verificadas las mallas curriculares en pregrado, de las universidades que ofertan la carrera de contabilidad y auditoría, se determinó que antes del año 2016, el $7 \%$ de las carreras, si incluyeron en su malla curricular la asignatura de auditoría forense; en tanto que el $93 \%$ restante, no consideraron como parte del programa a la indicada materia. Posterior al año 2016, con la formulación de los nuevos diseños de la carrera, y con el cambio de la nomenclatura del título de la carrera denominado; Licenciado en contabilidad y auditoría aprobado por el CES, se determinó que el $57 \%$ de los currículos incluyen la materia de auditoria forense, mientras que el $42 \%$ de las carreras no consideraron dicha asignatura en la malla curricular, evidenciándose por lo tanto, un importante incremento de los conocimientos, competencias y destrezas de la auditoría forense, a los futuros profesionales, vislumbrándose una mayor acogida en los esfuerzos y lucha contra la corrupción.

En el Ecuador antes y después del año 2016, las IES que ofertan solamente programas de posgrados (3 IES) no han ofertado maestrías en contabilidad y auditoría. En los demás programas académicos no se encontró asignaturas o módulos de auditoría forense lo cual denota la falta de preocupación en la formación y especialización de profesionales en el campo de la auditoría forense.

Apenas tres de las IES que ofertan la carrera de contabilidad y auditoría, han dictado maestrías en contabilidad y auditoría, tendiente a especializar a los profesionales contadores-auditores, incluyendo en sus programas la materia de auditoría forense, situación que revela la poca importancia que se otorga a esta área de conocimiento en la perspectiva de coadyuvar en la lucha contra la corrupción.

Esta situación hace necesario que las carreras de contabilidad y auditoría hagan constar en sus planes de estudio la enseñanza de auditoría forense, aportando al proceso de formación profesional, para cubrir las demandas que atraviesa hoy en día el Ecuador.

\section{Conclusiones}

El crecimiento de las acciones de fraude y la corrupción en el Ecuador, han ocasionada una demanda cada vez más creciente de profesionales investigadores del fraude que tengan conocimientos especializados 
y cumplan con los estándares internacionales. Las respuestas a los hechos ilícitos, han encaminado al desarrollo de la auditoría forense como medio para combatir la corrupción, cuyo cometido fundamental es colaborar y acompañar a las organizaciones públicas y privadas en la identificación oportuna de los riesgos e indicadores de fraude, que poseen o podrían presentarse, compenetrándose en las investigaciones y en los procedimientos legales necesarios para entender los diferentes esquemas contables utilizados por los perpetradores de fraude.

La auditoría forense dentro de sus acciones de prevención y disuasión no es la única herramienta para combatir y minimizar la corrupción, pero sí constituye, con toda certeza una técnica que puede entregar un aporte muy valioso que permita a las entidades de control y justicia, luchar efectivamente contra ese fenómeno, coordinadamente con el ministerio público y los órganos judiciales (Castillo, Figueredo y Méndez, 2012).

En este marco las carreras de contabilidad y auditoría de las universidades del Ecuador y la labor docente deberían involucrarse en la transmisión de conocimientos incluyendo en sus respectivos currículos, la asignatura auditoría forense, procurando que los profesionales contribuyan en la lucha contra la corrupción.

Es menester, promover el desarrollo de la auditoría forense con el objeto ampliar los estudios e investigaciones, como una actividad especializada que investiga y obtiene evidencias suficientes para el juzgamiento y sanción de los responsables de los hechos de fraude.

Se evidencia a partir del año 2016, un incremento importante de la asignatura auditoría forense en los currículos en grado, situación que en posgrado contrariamente tiene un escaso nivel de aplicación y transmisión de conocimientos y por lo tanto de profesionales especializados en el tratamiento de la auditoría forense.

Por consiguiente, la actuación tanto de las IES, así como del poder judicial y fiscalía ha sido débil, demostrando su insuficiente capacidad de respuesta ante hechos fraudulentos que atenta la economía y el bienestar de la población más pobre, disminuyendo la confianza y credibilidad en los sistemas de justicia que se rinden en la lucha contra la corrupción. 


\section{Referencias bibliográficas}

Aidt, T. (2011). Corruption and sustainable development. En S. Rose-Ackerman y T. Søreide, International Handbook on the Economics of Corruption Volume Two (pp. 3-51). Northamp-ton: Edward Elgar.

Akers, R. L. (2013). La generalidad de la teoría del autocontrol: una primera extensión de la teoría general del delito a JOURNAL OF SCIENCE AND RESEARCH: REVISTA CIENCIA E INVESTIGACIOÓN, E-ISSN: 2528-8083, VOL. 2, NO. 7, JULIO - SEPTIEMBRE 2017, PP. 37-44 44 los países de habla hispana, volumen 28. Editorial Dykinson, SL.

Americas's Accountability Anti-Corruption Project. (2004). La Corrupción en América Latina: Estudio analítico basado en una revisión bibliográfica y entrevistas. Virginia: Respondanet.

Arellano, D. (2017). Corrupción como proceso organizacional: comprendiendo la lógica de la desnormalización de la corrup-ción. Contaduría y Administración, 62(3), 810-826. Recupera-do de http:// dx.doi.org/10.1016/j.cya.2016.01.005

Badillo, J. Auditoria forense fundamentos. Extraído el 14 de Septiembre, 2010 de http://212.9.83.4/auditoria/ home.nsf/Todos/BC491B739B8E649AC12572F00057A6B4/\$FILE/Auditoria+Forense_Fundamentos.pdf

Banco Mundial. (2009). Las múltiples caras de la corrupción. Aspectos vulnerables por sectores. Washington, D.C.: Banco Mundial.

Cano, M. (2006). Auditoría forense aplicada en investigaciones de corrupción administrativa. En Control interno y fraudes. Bogotá: Ecoe Ediciones.

Cano, M. A. y Lugo. D. (2005). Auditoría forense en la investigación criminal del lavado de dinero y activos. Bogotá: ECOE Ediciones.

Castillo, L., Figueredo, C. y Méndez, E. (2012). La Audito-ría Forense en América Latina (casos Colombia, Venezuela y Ecuador). Gestión y Gerencia, 6(1), 26-46. Recuperado de ht-tps://dialnet.unirioja.es/servlet/ articulo?codigo=5303207 CEPAT. (2005). Auditoría Forense,

De La Torre, M. (2016). Lavado de Activos: Situación actual del Ecuador frente al GAFI. Revista Publicando, 3(8), 317-334. Recuperado de http://www.rmlconsultores.com/revista/index. php/crv/article/view/147

De La Torre, M. (2017). Responsabilidad Social Corporativa y Auditoría Interna, una exigencia global imperativa. Revista Metanoia, 3(3), 113-126.

Estupiñán, R. (2006). Control interno y fraudes. Bogotá: Ecoe Ediciones.

Herramienta de las EFS en la lucha contra la corrupción. En XV Asamblea General de la Organización 
Latinoamericana y del Caribe de Entidades Fiscalizadoras Superiores (OLACEFS). Recuperado de https:// www.ecoeediciones.com/wp-content/uploads/2015/07/Control-interno-y-fraudes-3ra-Edicio\%CC\%81n.pdf

Instituto de Auditores Internos del Ecuador (2013). Guía para la Práctica: Auditoría Interna y Fraude.

Lugo, Danilo, Auditoría Forense una perspectiva de investigación científica, United States InterAmerican Community Affairs, 2005. Badillo, J. (2005). Auditoría Forense. URL: www.isaca.org.ec/docdown/.

Ocampo, C., Trejos, O. y Solarte, G. (2010). Las técnicas forenses y la Auditoría. Scientia Et Technica 16(45), 108-113. Recuperado de http://www.redalyc.org/pdf/849/84917249019. pdf

Paiva Q. W. (2011) Control Interno Fraude y Corrupción. Enfoque de Auditoria Forense. Editorial Educación y Cultura. Cochabamba - Bolivia.

Ramírez, A. E. L. and Piedrahita, V. S. (2012). La auditoría forense: un campo en potencia. Adversia, (8):55-66.

Ríos Boyan, O. R. (2014). Auditoria forense fraudes contables y delitos de cuello blanco. Revista Ventana Cientifica, page 60.

Ríos, O. (2014). Auditoría Forense fraudes contables y delitos de cuello blanco. Ventana Científica 1(7), 60-63. Recuperado de http://www.revistasbolivianas.org.bo/pdf/rvc/v1n7/v1n7_ a08.pdf

Rocha Ch. J. (2009) Auditoria Forense, Técnicas de Detección Investigación de Fraudes e Irregularidades. Ediciones SIMMER. Santa Cruz - Bolivia

Rodríguez, L. A. L., Patiño, E. R., and Garzón, R. E. G. (2010). Auditoría forense: los procedimientos del contador público en la investigación del fraude corporativo. Gestión \& Sociedad, 3(2):141-160.

Roy A. (2007). Corruption in business: management theory and practice, Int. J. Business and Globalisation, 1(3), 311-327. Re-cuperado dehttp://dx.doi.org/10.1504/IJBG.2007.015051.

Rozas Flores, A. (2009). Auditoria forense "forensic audit". Revista de la Facultad de Ciencias Contables, 16(32):73- 101. 